\title{
Tight-binding molecular-dynamics study of liquid Si
}

\author{
C. Z. Wang \\ Ames Laboratory, Ames, Iowa 50011 \\ and Department of Physics and Microelectronics Research Center, Iowa State University, Ames, Iowa 50011 \\ C. T. Chan \\ Ames Laboratory, Ames, Iowa 50011 \\ K. M. Ho \\ Ames Laboratory, Ames, Iowa 50011 \\ and Department of Physics and Microelectronics Research Center, Iowa State University, Ames, Iowa 50011
}

(Received 9 October 1991)

\begin{abstract}
We have carried out molecular-dynamics simulation of liquid Si using the tight-binding Hamiltonian proposed by Goodwin, Skinner and Pettifor. The simulation results on the structural and dynamical properties (radial and angular distribution functions, the electronic density-of-states, and the diffusion constant) are presented and compared with results from classical-potential simulation and $a b$ initio molecular dynamics (Car-Parrinello method).
\end{abstract}

\section{INTRODUCTION}

Unlike most metallic liquids, liquid $\mathrm{Si}$ is found to have a coordination number between 6 and 7 (Refs. 1 and 2) rather than 10-12. This low coordination number indicates that the strong directional bonding that stabilizes the solid in the diamond crystal structure is still quite important in the liquid state. With the development of simulation techniques in the past two decades, there have been many studies of the properties of various liquid systems. However, an accurate simulation of the properties of liquid $\mathrm{Si}$ is still a challenging problem because of the difficulty in devising a simple interatomic potential capable of describing correctly the directional nature of the covalent bonding present in Si. Among the many empirical classical potentials ${ }^{3-9}$ proposed for calculating the properties of Si, only the Stillinger-Weber (SW) potential ${ }^{3}$ gives a qualitatively correct description for the properties of the liquid state $;^{3,10,11}$ however, the accuracy is still far from being satisfactory. On the other hand, the recently developed $a b$ initio molecular-dynamics scheme [the socalled Car-Parrinello (CP) method] (Ref. 12) made it possible to calculate the properties of liquid $\mathrm{Si}$ at finite temperature within the density-functional formalism with local-density approximation ${ }^{13}$ (LDA) which was shown to give a very good description of the energetics of $\mathrm{Si}$ in the crystalline phases. The method at present is computationally too expensive for extensive simulations. However, the results are very useful as a benchmark to test the accuracies of more approximate approaches.

Recently, we explored the possibility of molecular dynamics with a tight-binding Hamiltonian for studying such complex systems. In this "tight-binding moleculardynamics" (TBMD) approach, the electronic structure effects are incorporated into molecular dynamics through a parametrized tight-binding Hamiltonian. The covalent bonding of the material enters the calculations in a natural way from the underlying electronic structure rather than through ad hoc $N$-body potentials. Unlike the CarParrinello scheme, which relies on expansion of the electronic wave functions by plane waves, ${ }^{12}$ the tight-binding electronic calculations require only a few atomic orbitals for each atom, allowing a larger number of atoms and longer simulation periods to be tackled within the present computer capabilities. In addition, in our TBMD approach, the electronic degrees of freedom do not enter explicitly into the dynamics of the simulation, thus, we do not have the problem of the heating up of the electron system encountered in the Car-Parrinello method.

A brief description of the present work has been previously reported. ${ }^{14}$ The purpose of this paper is to provide a more comprehensive description of the details of the calculational method and the simulation results. In this paper, we first report studies of the melting behavior of crystalline Si. Results on the melting temperature as well as the latent heat of fusion are presented. We then studied the detailed structural and dynamical properties of the liquid state. Results on the radial and angular distribution functions, the electronic density of states, and the diffusion constant are presented and compared with results from classical-potential simulations and $a b$ initio molecular-dynamics (CP method) simulations. Virkkunen, Laasonen, and Nieminen have independently presented results on a similar study of the properties of liquid $\mathrm{Si},{ }^{15}$ also using the tight-binding potential of Goodwin et al. The results from both calculations are very similar.

The rest of the paper is arranged as follows: In Sec. II, we describe the TBMD scheme and the tight-binding potential model for Si. The details of the MD simulation along with the results are presented in Sec. III. Finally, we conclude our paper by a brief summary in Sec. IV. 


\section{TIGHT-BINDING MOLECULAR-DYNAMICS SCHEME}

Details of the tight-binding molecular-dynamics scheme have been given in earlier publications. ${ }^{16}$ In TBMD simulations, the atomic motions are governed by the following Hamiltonian:

$$
H=\sum_{i} \frac{P_{i}^{2}}{2 m}+\sum_{n, k}^{\text {occupied }}\left\langle\psi_{n k}\left|H_{\mathrm{TB}}\right| \psi_{n k}\right\rangle+E_{\mathrm{rep}},
$$

where the first term is the kinetic energy of the ions, the second term is the quantum-mechanical bond energy calculated from the sum of the occupied electronic eigenvalues obtained from a parametrized tight-binding Hamiltonian $H_{\mathrm{TB}}$, and the third term is a short-ranged repulsive potential. The electronic degrees of freedom are explicitly involved in the force calculation but not in the dynamics. This feature allows the interatomic interactions to be characterized by quantum mechanics, while the motion of the atoms is governed by classical mechanics. The time steps for solving the equation of motions are similar to those used in traditional moleculardynamics simulations using classical potentials.

In the present simulation, a tight-binding Hamiltonian for Si proposed by Goodwin, Skinner, and Pettifor ${ }^{17}$ was used. The model uses a minimal basis of $s p^{3}$ orbitals to construct the tight-binding Hamiltonian $H_{\mathrm{TB}}$ for the electronic structure calculations, namely,

$$
H_{\mathrm{TB}}=\sum_{i} \sum_{\alpha=s, p} \varepsilon_{\alpha} a_{i \alpha}^{\dagger} a_{i \alpha}+\sum_{i, j} \sum_{\alpha, \beta=s, p} h_{i \alpha, j \beta} a_{i \alpha}^{\dagger} a_{j \beta} .
$$

There are two on-site parameters and four two-center hopping parameters in $H_{\mathrm{TB}}: \varepsilon_{s}-\varepsilon_{p}=8.295 \mathrm{eV}$, $h_{s s \sigma}\left(r_{0}\right)=-1.82 \mathrm{eV}, h_{s p \sigma}\left(r_{0}\right)=1.96 \mathrm{eV}, h_{p p \sigma}\left(r_{0}\right)=3.06$ $\mathrm{eV}$, and $h_{p p \pi}\left(r_{0}\right)=-0.87 \mathrm{eV}$, where $r_{0}=2.35 \AA$ is the nearest-neighbor distance in the diamond structure.

Unlike the usual $r^{-2}$ scaling ${ }^{18,19}$ used in most tightbinding models, in the present model the hopping parameters between two sites separated by distance $r$ are scaled as

$h_{\lambda}(r)=h_{\lambda}\left(r_{0}\right)\left(\frac{r_{0}}{r}\right)^{2} \exp \left\{2\left[-\left(\frac{r}{r_{c}}\right)^{n_{c}}+\left(\frac{r_{0}}{r_{c}}\right)^{n_{c}}\right]\right\}$,

where the subscript $\lambda$ denotes the four possible types of interatomic hopping, i.e., ss $\sigma, s p \sigma, p p \sigma$, and $p p \pi$, $r_{c}=3.67 \AA$ and $n_{c}=6.48$ are scaling parameters.

The repulsive potential $E_{\text {rep }}$ consists of a sum of pairwise interactions $\phi(r)$ between $\mathrm{Si}$ atoms,

$$
\begin{aligned}
\phi(r)= & \phi\left(r_{0}\right)\left[\frac{r_{0}}{r}\right]^{4.54} \\
& \times \exp \left\{4.54\left[-\left(\frac{r}{r_{c}}\right]^{n_{c}}+\left(\frac{r_{0}}{r_{c}}\right)^{n_{c}}\right]\right\},
\end{aligned}
$$

where $\phi\left(r_{0}\right)=3.4581 \mathrm{eV}$, and $r_{c}$ and $n_{c}$ take the same values as above.

This tight-binding model is similar to the earlier formulation proposed by Chadi ${ }^{18}$ and by Harrison. ${ }^{10}$ The distance dependence of the tight-binding hopping parameters and the pair potential have been rescaled so that the model reproduces the first-principles-calculated $\mathrm{Si}$ bulk phase diagram (see Fig. 1) when the tight-binding hopping parameters are restricted to the nearest neighbors. When the model is used for simulating the liquid state, it is necessary to choose a unique cutoff distance for the interatomic interactions. However, we note that it is impossible to obtain a cutoff distance that satisfies the condition that all the crystalline structures of $\mathrm{Si}$ have only nearest-neighbor interactions. In this study, we choose $3.60 \AA$, which is between the nearest- and next-nearestneighbor distances of diamond structure, as the cutoff distance. This choice does not alter the energy curve of the diamond structure near the equilibrium volume but does have notable effects on those of the metallic structures. In comparison with the original nearest-neighbor scheme, the cohesive energy and equilibrium volume of the metallic phases have been shifted toward higher values as one can see from Fig. 1(c). The consequence of



FIG. 1. Cohesive energy as a function of relative volume $V / V_{0}\left(V_{0}\right.$ is the equilibrium volume of the diamond structure) for several bulk phases of $\mathrm{Si}$ (as indicated). (a) The results of LDA calculations (Ref. 20); (b) the results of TB calculations using the parameters of Ref. 17 and with nearest-neighbor interactions only; (c) the results of the TB calculation using the parameters of Ref. 17 but with a unique interaction cutoff of $3.60 \AA$. See also text for details. 
TABLE I. $T=0$ equilibrium properties of silicon obtained by the present tight-binding model are compared with the results obtained by first-principles (LDA) calculation and by the SW potential and with experiment. $C_{44}^{0}$ denotes the shear constant that would appear in the absence of internal displacements. $C_{44}$ is the shear constant in the absence of internal stress. The firstprinciples results are taken from Refs. 21 and 22 . The SW potential results are taken from Refs. 23 and 24. The experimental data are quoted from Ref. 25.

\begin{tabular}{lrrrr}
\hline \hline & TB & LDA & SW & Expt. \\
\hline$B\left(10^{11} \mathrm{erg} / \mathrm{cm}^{2}\right)$ & 10.42 & $9.20^{\mathrm{a}}$ & $10.14^{\mathrm{c}}$ & 9.78 \\
$C_{11}-C_{12}\left(10^{11} \mathrm{erg} / \mathrm{cm}^{3}\right)$ & 6.99 & $9.80^{\mathrm{b}}$ & $7.50^{\mathrm{c}}$ & 10.12 \\
$C_{44}^{0}\left(10^{11} \mathrm{erg} / \mathrm{cm}^{3}\right)$ & 12.24 & $11.10^{\mathrm{b}}$ & & \\
$C_{44}\left(10^{11} \mathrm{erg} / \mathrm{cm}^{3}\right)$ & 6.85 & $8.50^{\mathrm{b}}$ & $5.64^{\mathrm{c}}$ & 7.96 \\
$v_{\mathrm{LTO}(\Gamma)}(\mathrm{THz})$ & 18.31 & $15.16^{\mathrm{a}}$ & $17.83^{\mathrm{c}}$ & 15.53 \\
$v_{\mathrm{TA}(X)}(\mathrm{THz})$ & 4.50 & $4.45^{\mathrm{a}}$ & $5.96^{\mathrm{c}}$ & 4.49 \\
$v_{\mathrm{TO}(X)}(\mathrm{THz})$ & 16.38 & $13.48^{\mathrm{a}}$ & & 13.90 \\
$v_{\mathrm{LO} A(X)}(\mathrm{THz})$ & 13.18 & $12.16^{\mathrm{a}}$ & & 12.32 \\
$\gamma_{\mathrm{LTO}(\Gamma)}$ & 0.92 & $0.92^{\mathrm{a}}$ & $0.80^{\mathrm{d}}$ & 0.98 \\
$\gamma_{\mathrm{TA}(X)}$ & -1.29 & $-1.50^{\mathrm{a}}$ & $-0.04^{\mathrm{d}}$ & -1.40 \\
$\gamma_{\mathrm{TO}(X)}$ & 1.16 & $1.34^{\mathrm{a}}$ & $0.89^{\mathrm{d}}$ & 1.50 \\
$\gamma_{\mathrm{LOA}(X)}$ & 0.95 & $0.92^{\mathrm{a}}$ & $0.83^{\mathrm{d}}$ & 0.90 \\
\hline \hline
\end{tabular}

${ }^{\mathrm{a}}$ Reference 21.

${ }^{\text {b Reference } 22 .}$

${ }^{\mathrm{c}}$ Reference 23.

${ }^{\mathrm{d}}$ Reference 24 .

these effects will be discussed in the next section when the simulation results are presented.

Prior to the molecular-dynamics simulations of liquid $\mathrm{Si}$, we have examined the tight-binding model by applying it to study the ground-state $(T=0)$ properties of crystalline Si. The results, listed in Table I, indicate that the model gives a good description of the elastic, vibrational, as well as anharmonic properties of $\mathrm{Si}$ in the diamond structure.

\section{SIMULATION DETAILS AND RESULTS}

Constant-volume and constant-temperature molecular-dynamics simulations are performed with a 64-Siatom cubic cell and with periodic boundary conditions to compare with the results of recent $a b$ initio moleculardynamics simulations. ${ }^{13}$ The time step used in our simulation is $1.07 \times 10^{-15} \mathrm{~s}$. The density of the sample is set equal to the experimental density at the triple point, i.e., $2.53 \mathrm{~g} / \mathrm{cm}^{3}$.

We begin the simulation with the system in the diamond structure and observe the melting of the crystal upon raising the temperature. The stochastic collision method proposed by Andersen ${ }^{26}$ is used to control the temperature of the system. Due to the finite size of the unit cell and short simulation time ( $\sim 2.5 \mathrm{ps})$, superheating is observed and the crystal melts at a temperature higher than the experimental melting temperature of $T=1685 \mathrm{~K}$. For the same reason, when we cool the hot liquid down to $T=1300 \mathrm{~K}$, the system maintains the liquid structure. In Fig. 2, the total energy of the system is plotted as a function of temperature as we heat the system from the crystalline state and cool it from the hot

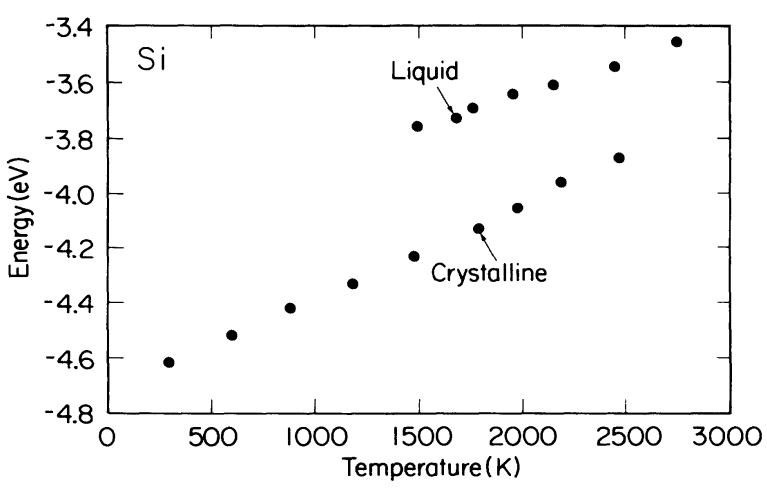

FIG. 2. Total energy (per atom) as a function of temperature for $\mathrm{Si}$ in the crystalline and liquid phases.

liquid state. The data have been averaged over $1500 \mathrm{MD}$ steps after 1000 steps of thermal equilibration at each temperature. We see clearly that the energy of the crystalline branch and the energy of the liquid branch are separated by a gap that gives the latent heat of fusion for the system. At $T \sim 1700 \mathrm{~K}$, the energy gap between the two branches is found to be about $0.43 \mathrm{eV}$, which is close to the experimental value of the latent heat $[0.47 \mathrm{eV}$ at $T \approx 1685 \mathrm{~K}$ (Ref. 27)] and smaller than the value of $\sim 0.54 \mathrm{eV}$ predicted by the Stillinger-Weber potential. ${ }^{3}$

The structural and electronic properties of liquid $\mathrm{Si}$ have been studied at $T=1780 \mathrm{~K}$, which is close to the experimental triple point. The liquid state is obtained by cooling down from hot liquid at $2700 \mathrm{~K}$. The cooling is performed in steps through 2500,2200 , and $2000 \mathrm{~K}$, with 2500 MD steps for each temperature and using Andersen's method for the temperature control. At 1780 K, 6000 MD steps with Andersen's method for temperature control followed by another $6000 \mathrm{MD}$ steps with the traditional velocity-scaling method (with a tolerance of $180 \mathrm{~K}$ ) for temperature control have been performed to thermalize the sample. After these thermalization steps, the temperature of the system is very stable. We then used the following $8000 \mathrm{MD}$ steps (corresponding to $\sim 8.6 \mathrm{ps}$ ) to perform the statistical averages for the properties of interest. Although we maintained the velocityscaling temperature control throughout the statistical averaging steps, the thermostat is activated only about once in every $100 \mathrm{MD}$ steps on the average.

The result of the radial distribution function $g(r)$ is shown in Fig. 3 in comparison with that obtained by the Car-Parrinello method. The present $g(r)$ (thick line) exhibits a sharp nearest-neighbor peak and has an oscillatory tail about the value of 1 . This shape is very similar to that obtained by the $a b$ initio Car-Parrinello simulation (thin line). Integration of $g(r)$ up to its first minimum $\left(r_{m}=3.22 \AA\right)$ yields a coordination number of 7.17. This number is slightly larger than the experimental value of 6.4 (Refs. 1 and 2) and the Car-Parrinello simulation result of $6.5,{ }^{13}$ but smaller than the coordination number of 8.07 predicted by the Stillinger-Weber potential. ${ }^{3}$ The coordination number obtained by us is also slightly larger than the value of 6.4 reported by Virkkunen, Laasonen, and Nieminen ${ }^{15}$ who used essentially the same tight- 


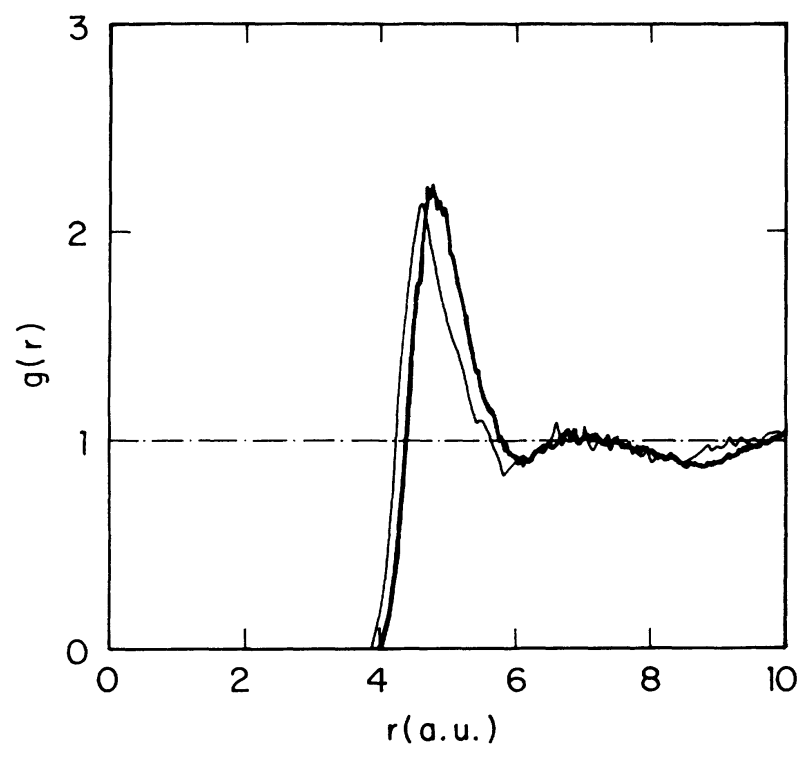

FIG. 3. Radial distribution function of liquid Si. The thick line represents the present TBMD result. The thin line is the result obtained by the Car-Parrinello method (Ref. 13).

binding model with a slightly different scaling parameter $r_{c}(3.67 \AA$ in this paper versus $3.6 \AA$ in Ref. 15). Since the original tight-binding model of Goodwin, Skinner, and Pettifor ${ }^{17}$ does not define a unique cutoff distance, it is possible that the use of the model with a different cutoff in the molecular-dynamics simulation may also yield slightly different results. The coordination number is also sensitive to the location of first minimum in $g(r)$ and may be subjected to some error due to statistical fluctuations from the simulation. In a previous work, ${ }^{14}$ we have shown that the integration of $g(r)$ obtained from our simulation to $3.12 \AA$ (the upper limit of integration for the Car-Parrinello results) would give a coordination number of 6.5. We note that the first peak in $g(r)$ obtained from the present TBMD simulation and from the Stillinger-Weber potential ${ }^{3}$ is slightly shifted towards larger distances with respect to that obtained by the CarParrinello scheme. The average nearest-neighbor distance from our simulation is $\sim 2.54 \AA$, which is close to $2.56 \AA$ given by the SW potential but slightly larger than $\sim 2.46 \AA$ obtained by the Car-Parrinello simulation ${ }^{13}$ and $2.50 \AA$ obtained by Virkkunen, Laasonen, and Nieminen. ${ }^{15}$ The experimental value is $2.50 \AA .{ }^{1,2}$ We also note that, within the given density and temperature in the present TBMD simulation, the pressure as estimated through the virial theorem is found to be $\sim 75 \mathrm{kbar}$, which is apparently a bit too large. Since the equilibrium volume of the diamond structure calculated from the present tight-binding model is in good agreement with that obtained by the LDA calculation, the fact that the present tight-binding model tends to predict larger equilibrium bond lengths for higher coordinated structures [see Fig. 1(c)] may be responsible for the overestimation of the average interatomic distance and pressure in the liquid state.

In Fig. 4, the TBMD results of bond-angle distribution functions are presented in comparison with the Car-
Parrinello results. With a bond-length cutoff at $r=2.51$ $\AA$ (2.40 $\AA$ in the Car-Parrinello simulation), which is close to the nearest-neighbor distance of $\mathrm{Si}$ in the diamond structure, only a single peak around $100^{\circ}$ is found. This suggests that short-ranged tetrahedral ordering persists in the liquid phase in Si. While with a bond-length cutoff at $r=3.22 \AA$, i.e., the first minimum at $g(r)$, the bond-angle distribution function shows two peaks, one around $60^{\circ}$ and the other around $100^{\circ}$. This double-peak feature reflects the fact that both tetrahedral bonded and higher coordinated atoms are contributing to the nearest-neighbor peak in $g(r)$. These results are similar to those obtained by Virkkunen, Laasonen, and Nieminen, ${ }^{15}$ and are in very good agreement with the CarParrinello simulation results. ${ }^{13}$ We note that the SW potential gives qualitatively different results: it fails to pre-
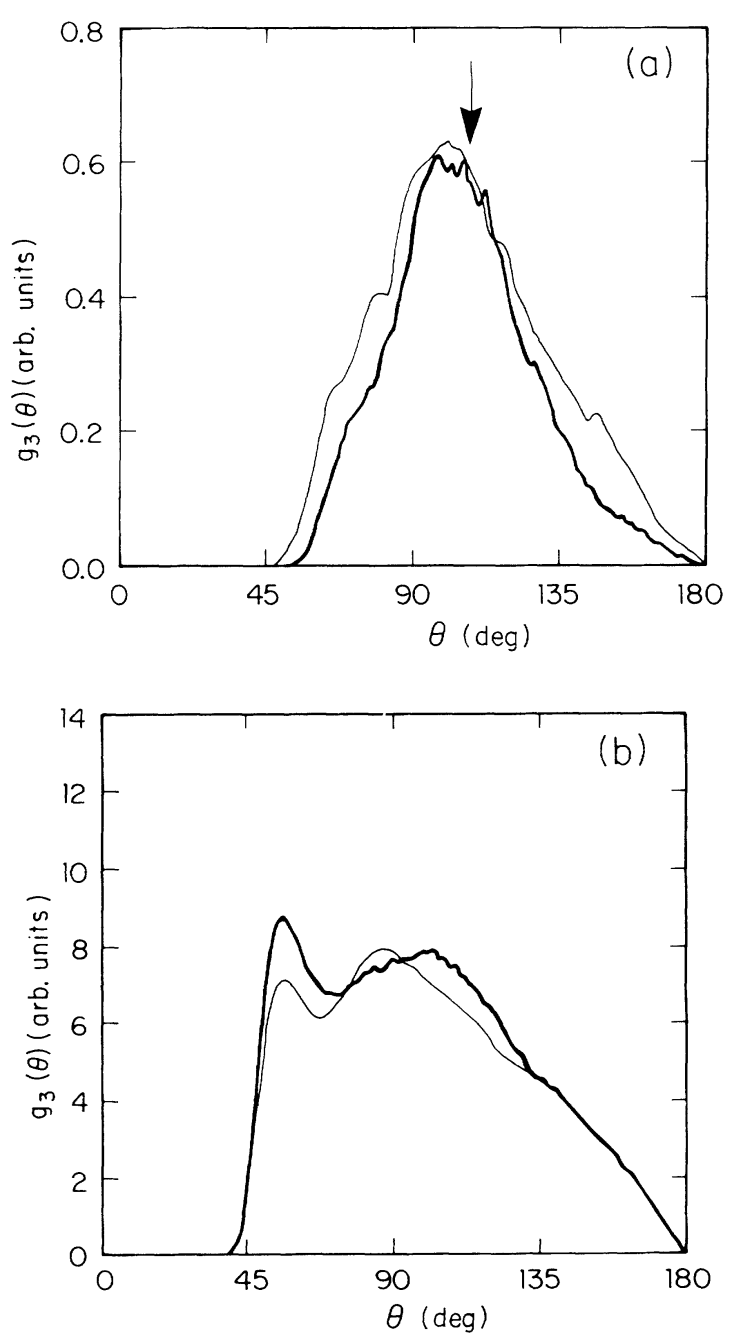

FIG. 4. Bond-angle distribution functions of liquid Si for (a) bond lengths less than $2.51 \AA(2.40 \AA$ in the Car-Parrinello simulation) and (b) bond lengths less than $3.22 \AA$ ( $3.12 \AA$ in the Car-Parrinello simulation). The thick line represents the present TBMD result and the thin line is the result obtained by the Car-Parrinello method (Ref. 13). The arrow indicates the position of the tetrahedral angle. 


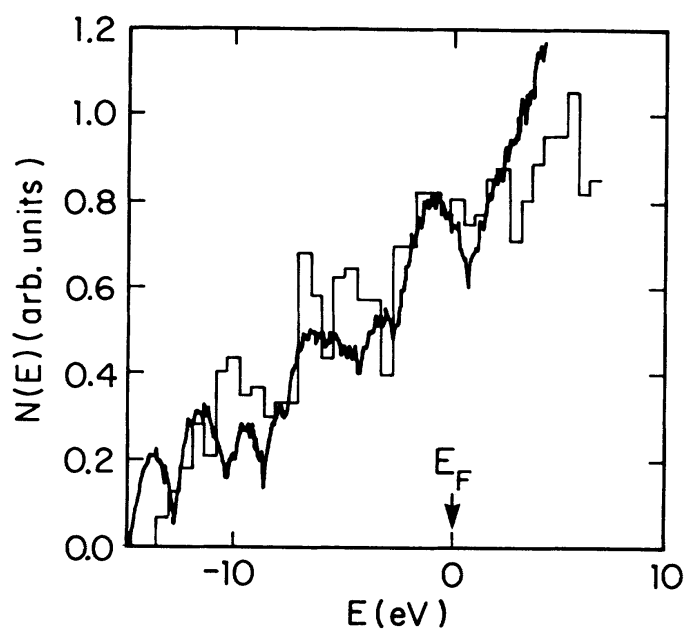

FIG. 5. Electronic density of states of liquid Si. The thick line represents the present TBMD result and the thin line is the result obtained by the Car-Parrinello method (Ref. 13).

dict a second peak at $60^{\circ}$ besides the main peak at the tetrahedral bond angle. ${ }^{13,15,28}$

One of the advantages of the TBMD scheme over the classical-potential scheme is that it can also provide information on the electronic properties. In Fig. 5, the electronic density of states averaged over all useful MD steps is plotted in comparison with the Car-Parrinello result. The absence of a gap at the Fermi energy indicates that liquid $\mathrm{Si}$ is metallic.

The dynamical properties of liquid Si have been investigated by calculating the atomic mean-square displacement,

$$
\left\langle R^{2}(t)\right\rangle=\left\langle N^{-1} \sum_{i=1}^{N}\left|\mathbf{R}_{i}(t+\tau)-\mathbf{R}_{i}(\tau)\right|^{2}\right\rangle_{\tau} .
$$

The data used for calculating the $\left\langle R^{2}(t)\right\rangle$ are collected at every $10 \mathrm{MD}$ steps over 8000 steps (corresponding to 8.6 ps). Initial states $\left[\mathbf{R}_{i}(\tau)\right]$ in the first half of the data points one used to perform the average denoted by the brackets. The result of $\left\langle R^{2}(t)\right\rangle$ as a function of time $t$ is plotted in Fig. 6. By using the Einstein relation, $D=\lim _{t \rightarrow \infty}\left[\left\langle R^{2}(t)\right\rangle / 6 t\right]$, we deduce the diffusion constant for liquid $\mathrm{Si}$ to be $1.25 \times 10^{-4} \mathrm{~cm}^{2} / \mathrm{s}$ at $1780 \mathrm{~K}$, which is close to $1.1 \times 10^{-4} \mathrm{~cm}^{2} / \mathrm{s}$ at $1740 \mathrm{~K}$ obtained by Virkkunen, Laasonen, and Nieminen. Our result is smaller than the Car-Parrinello simulation result of $2.26 \times 10^{-4} \mathrm{~cm}^{2} / \mathrm{s}$ at $1800 \mathrm{~K},{ }^{13}$ but larger than $0.694 \times 10^{-4} \mathrm{~cm}^{2} / \mathrm{s}$ at $1691 \mathrm{~K}$ (Ref. 10) and $0.98 \times 10^{-4}$ $\mathrm{cm}^{2} / \mathrm{s}$ at $2010 \mathrm{~K}$ (Ref. 11) predicted by the $\mathrm{SW}$ potential.



FIG. 6. Mean-square displacement of liquid $\mathrm{Si}$ as a function of time at $T=1780 \mathrm{~K}$.

It is possible that the fact the liquid state is under compression (pressure $\sim 75 \mathrm{kbar}$ ) may be responsible for the slower diffusion in the present simulations.

\section{SUMMARY}

In summary, we have studied the properties of liquid $\mathrm{Si}$ with a tight-binding molecular-dynamics scheme. In comparison with the simulations using the classical Stillinger-Weber potential, our simulation results are in significantly better agreement with results from $a b$ initio calculations and experiments. We note that there are some subtle differences between the results of the present TBMD and those of the Car-Parrinello simulation. These small differences may be resolved by further slight modifications of the tight-bonding model. We now have a working scheme that is comparable in accuracy to $a b$ initio molecular dynamics, yet hundreds of times faster. This scheme will be useful for studying more complex $\mathrm{Si}$ systems such as Si clusters and amorphous Si. Phenomena like atomic diffusion, which demand long simulation times for the extraction of meaningful results, can also be addressed by the present scheme.

\section{ACKNOWLEDGMENTS}

This work is supported by the Director of Energy Research, Office of Basic Energy Sciences and by the U.S. Air Force Office of Scientific Research. Ames Laboratory is operated for the U.S. Department of Energy by Iowa State University under Contract No. W-7405ENG-82.
${ }^{1}$ Y. Waseda and K. Suzuki, Z. Phys. B 20, 339 (1975).

2J. P. Gabathuler and S. Steeb, Z. Naturforsch. Teil A 34, 1314 (1979).

${ }^{3}$ F. H. Stillinger and T. A. Weber, Phys. Rev. B 31, 5262 (1985).

${ }^{4}$ J. Tersoff, Phys. Rev. Lett. 56, 632 (1986); Phys. Rev. B 37, 6991 (1988).
${ }^{5}$ R. Biswas and D. R. Hamann, Phys. Rev. Lett. 55, 2001 (1985). ${ }^{6}$ B. W. Dodson, Phys. Rev. B 35, 2795 (1987).

${ }^{7}$ J. R. Chelikowsky, J. C. Phillips, M. Kamal, and M. Strauss, Phys. Rev. Lett. 62, 292 (1989).

${ }^{8}$ M. I. Baskes, Phys. Rev. Lett. 59, 2666 (1987).

${ }^{9}$ K. E. Khor and Das Sarma, Phys. Rev. B 38, 3318 (1988). 
${ }^{10}$ J. Q. Broughton and X. P. Li, Phys. Rev. B 35, 9120 (1987).

${ }^{11}$ P. Allen and J. Broughton, J. Phys. Chem. 91, 4964 (1987).

${ }^{12}$ R. Car and M. Parrinello, Phys. Rev. Lett. 60, 204 (1988).

${ }^{13}$ I. Štich, R. Car, and M. Parrinello, Phys. Rev. Lett. 63, 2240 (1989); Phys. Rev. B 44, 4262 (1991).

${ }^{14}$ C. Z. Wang, C. T. Chan, and K. M. Ho, Phys. Rev. Lett. 66, 189 (1991).

${ }^{15}$ R. Virkkunen, K. Laasonen, and R. M. Nieminen, J. Phys: Condens. Matter 3, 7455 (1991).

${ }^{16}$ C. Z. Wang, C. T. Chan, and K. M. Ho, Phys. Rev. B 39, 8592 (1989); 40, 3390 (1989).

${ }^{17}$ L. Goodwin, A. J. Skinner, and D. G. Pettifor, Europhys. Lett. 9, 701 (1989).

${ }^{18}$ D. J. Chadi, Phys. Rev. Lett. 41, 1062 (1978); Phys. Rev. B 29, 785 (1984).

${ }^{19}$ W. A. Harrison, Electronic Structure and the Properties of Solids (Freeman, San Francisco, 1980).

${ }^{20}$ M. T. Yin and M. L. Cohen, Phys. Rev. B 26, 5668 (1982).
${ }^{21}$ M. T. Yin and M. L. Cohen, Phys. Rev. B 26, 3259 (1982).

${ }^{22}$ O. H. Nielsen and R. M. Martin, Phys. Rev. B 32, 3798 (1985).

${ }^{23}$ E. R. Cowley, Phys. Rev. Lett. 60, 2379 (1988).

${ }^{24}$ C. Z. Wang, C. T. Chan, and K. M. Ho, Phys. Rev. B 42, 11276 (1990).

${ }^{25}$ Semiconductors: Physics of Group IV Elements and III-V Compounds, edited by O. Madelung, M. Schulz, and $\mathrm{H}$. Weiss, Landolt-Börnstein, New Series, Group III, Vol. 17a (Springer-Verlag, Berlin, 1982); Semiconductors: Instrinsic Properties of Group IV Elements and III-V, II-VI and I-VII Compounds, edited by O. Madelung and M. Schulz, LandoltBörnstein, New Series, Group III, Vol. 22a (Springer-Verlag, Berlin, 1987).

${ }^{26}$ H. C. Andersen, J. Chem. Phys. 72, 2384 (1980).

${ }^{27}$ A. R. Ubbelohde, The Molten State of Matter (Wiley, New York, 1978), p. 239.

${ }^{28}$ W. D. Leudtke and U. Landman, Phys. Rev. B 37, 4656 (1988). 